\title{
Antioxidant and prooxidant activity of new 1,2-diols and thiadiazoles derivatives in Saccharomyces cerevisiae yeast cells
}

\author{
Arzu KARATEPE ${ }^{1^{*}}$ (D), Ahmet ÇETIN ${ }^{1}$ \\ ${ }^{1}$ Bingöl University, Department of Chemistry, Bingöl/ TURKEY
}

\section{Abstract}

The present research was undertaken to determine the effect of substituted 2,2'-[(2R,3R)-2,3dihydroxy-1,4-dioxobutane-1,4-diyl]bis(N-R-hydrazine-1-carbothioamide and (1R,2R)-1,2bis[5-(R-amino)-1,3,4-thiadiazole-2-yl]ethane-1,2-diols on the antioxidant status of the yeast Saccharomyces cerevisiae cells. This cell serves a good eukaryotic model system for the study of molecular mechanisms of oxidative stress. The Saccharomyces cerevisiae yeast cells were treated a series of 1,2-diols and thiadiazoles compounds and the malondialdehyde (MDA) and antioxidant vitamins (A, E, C) levels in the medium were measured by HPLC-UV. In the comparison done among groups, the MDA which is an indicator of lipid peroxidation and Vitamin E concentrations were showed statistically changed in the samples. Exposure of yeaset cells to L9 showed an increase in MDA and decrease in vitamin E levels but L2 and L8 showed decrease in MDA and increase in vitamin E levels. The results showed that compounds L9 caused a considerable oxidative stress and L2 and L8 have antioxidant activity.
\end{abstract}

\section{Article info}

History:

Received: 06.07.2020

Accepted: 25.08 .2020

Keywords:

1,2-diols, thiadiazoles,

MDA, vitamin,

saccharomyces

cerevisiae,

antioxidant.

\section{Introduction}

The natural or chemical materials such as pharmaceutics, dyes, organic materials and biologically active compounds are containing the nitrogen atom containing heterocycles [1]. The 1,3,4thiadiazole nucleus is well-known heterocyclic nuclei, which is a functional group of a variety of medicinal agents and natural products. Because of their diverse biological activity, the 1,3,4-thiadiazole groups have become great interest by the researchers as important class of heterocycles. These biological activities are including antimicrobial [2], antituberculosis [3], antioxidant [4], antiinflammatory [5], anticonvulsants [6], antidepressant and anxiolytic [7], antihypertensive [8], anticancer [9] and antifungal activity [10].

The diverse biological activities of the 1,3,4thiadiazole rings are associated with incorporating a toxophoric -N=C-S linkage of which has been showed in many pesticides [11-14] and therefore 1,3,4thiadiazoles and their Schiff bases have recently received significant importance [15]. Some natural products include enantiomerically pure 1,2-diols and this molecule is valuable intermediates in the organic synthesis of biologically active compounds [16]. They are readily transformed into chiral epoxides [17] aziridines, and amino alcohols [18]. Moreover, the1,2diol functionality is found in a number of synthetic and pharmaceutical intermediates [19].
The production of pharmaceuticals, chemical catalysts, and agrochemicals are need optically pure 1,2-diols [20]. Although the only commercial 1, 2, 4-thiadiazole drug is the antibiotic cefozopram, many drugs such asacetazolamide, methazolamide, megazol are containing 1,3,4-thiadiazole nucleus which are available in the market $[2,21]$.

As a result of these studies, it is suggested that 1,3,4thiadiazole derivatives have wide biological effects.

However, the nitrogen containing heterocyclic compounds becomes an important goal in modern organic synthesis for efficient antioxidant activity, nowadays.

The hydrogen peroxide, superoxide anions and hydroxyl radicals, which are known reactive oxygen species (ROS), are generated by aerobic life style and living cells are exposed to these molecules. Free radicals are highly reactive ions or molecules that have unpaired electron [22, 23]. In some special cases, if the amount of reactive oxygene species increases and the antioxidant capacity exceeds, oxidative stress occurs [1]. The extracellular xenobiotics and substances can be generating this stress condition by which cause the loss of cellular integrity [2]. The several lethal diseases such as cancer and neurodegenerative disorders as well as aging related with oxidative stress has been in great focus due to its correlation with a normal and inevitable eukaryotic process [3]. Reactive oxygen species (ROS) which are destroy to DNA via oxidative 
damage, have been associated many health problems such as coronary heart diseases and carcinogenesis $[24,25]$.

The complex physiological response contains both enzymes (e.g., catalase, superoxide dismutase) and protective molecules for resistance to oxidative stress $[22,23]$ and the non-enzymatic low molecular weight molecules such as ascorbate, tocopherols, flavonoids and glutathione are important in scavenging ROS [23].

The Saccharomyces cerevisiae yeast cells that possibility of using genetic approaches are made a common experimental model for studies of stress response at the molecular level. Mammalian and yeast cells activate a wide response involving several defense mechanisms which named adaptive response, to adapt sensitive cells for environmental change such as temperature shifts and increased ROS levels [26].

The aim of this work was to find antioxidant ability new chemical structures 1,2-diols and thiadiazoles by using Saccharomyces cerevisiae yeast cells.

\section{Materials and Methods}

\subsection{Materials and chemicals}

The vitamin A (all trans-retinol), MDA (1,1,3,3tetraethoxypropane for standart) were obtained from Sigma (St. Louis, MO, USA). Vitamin C (ascorbic acid), vitamin $\mathrm{E}$ ( $\alpha$-tocopherol), $\mathrm{HClO}_{4}$, methanol, $\mathrm{H}_{3} \mathrm{PO}_{4}$ and $\mathrm{KH}_{2} \mathrm{PO}_{4}$ were purchased from Merck (Darmtadt, Germany). Stock solutions of compounds were prepared as $5000 \mu \mathrm{M}$ solutions in DMSO (dimethylsulfoxid).

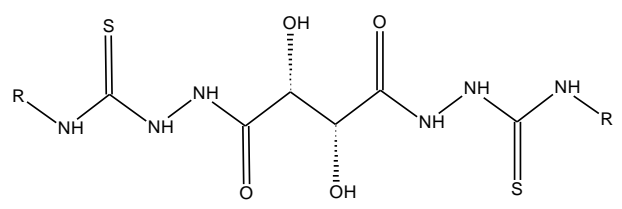

a

\subsection{Applied cells}

Saccharomyces cerevisiae yeast cells were used to determine antioxidant activity. Saccharomyces cerevisiae, a member of the fungi kingdom, is a singlecelled microorganism. Saccharomyces cerevisiae is ascomycetic yeast. The yeasts are contain high vitamin level, therefore increases its value as a nutrient. These cells are often used as a model for molecular responses to oxidative stress metabolism [26].

The dry yeast sample contining the Microorganism Saccharomyces cerevisiae was stored at $+4^{\circ} \mathrm{C}$ during the study. Preparation, cultivation of microorganism culture and the addition and incubation of chemicals to investigate the effect on microorganism were done in the Microbiology Laboratory. For Saccharomyces cerevisiae development and reproduction to be used in the experiment, Malt Extract Broth (Difco) was inoculated into and incubated for $48 \mathrm{~h}$. at $25 \pm 1{ }^{\circ} \mathrm{C}$. The prepared yeast, broth culture is inoculated into YEDP ( $1 \mathrm{~g}$ yeast extract for $100 \mathrm{ml}, 2 \mathrm{~g}$ bactopeptone, $2 \mathrm{~g}$ glucose, $2 \mathrm{~g}$ agar) into the medium at a rate of $1 \%$ $\left(10^{4}\right.$ yeast $\left./ \mathrm{ml}\right)$ at $25 \pm 0.1^{\circ} \mathrm{C}$ for $48 \mathrm{~h}$. has been incubated.

\subsection{Chemical compounds used}

The whole of the work will be carried out in the chemistry department of Firat University biochemistry and microbiology cell culture laboratory. The structure of the compounds 2,2'-[(2R,3R)-2,3-dihydroxy-1,4dioxobutane-1,4-diyl]bis(N-R-hydrazine-1-

carbothioamide and (1R,2R)-1,2-bis[5-(R-amino)1,3,4-thiadiazole-2-yl]ethane-1,2-diol to be used in the studies is given below [27].

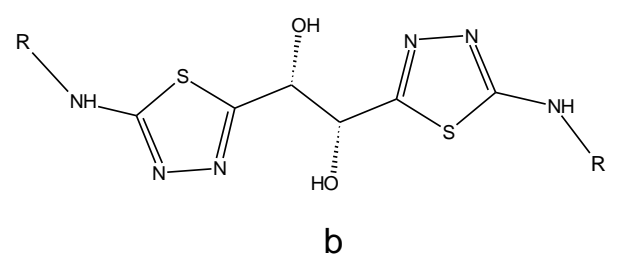

Figure 1. Structure of compounds a) 1,2-diols and b) thiadiazoles R: L1,L6 phenyl, L2,L7 p-tolyl, L3,L8 p-methoxyphenyl, L4,L9 allyl and L5,L10 ethyl.

\subsection{Test compounds treatmant Saccharomyces cerevisiae yeast cells for antioxidant prooxidant activity}

Saccharomyces cerevisiae yeast cells were added in test tubes at a density of about $1 \times 10^{6} \mathrm{ml}^{-1}$ cell, and incubated for 1 day before the experiment. The test compounds were prepared at specific concentration by dissolving in DMSO. The final concentrations of test compounds in cells were $50 \mu \mathrm{M}$ and incubated and stirred $24 \mathrm{~h}$. 


\subsection{Determination of MDA and vitamin $\mathrm{C}$}

MDA and vitamin $C$ levels were assayed according to the method of Karatepe, small modifications [28]. Briefly, the cells were collected and were gently rinsed twice with $2 \mathrm{ml}$ of ice-cold Krebs-Ringer-Hepes buffer at the end of the incubation period $(128 \mathrm{mM} \mathrm{NaCl}, 20$ $\mathrm{mM}$ Hepes, $1.4 \mathrm{mM} \mathrm{MgSO} 4,1.4 \mathrm{mM} \mathrm{CaCl}_{2}, 1 \mathrm{mM}$

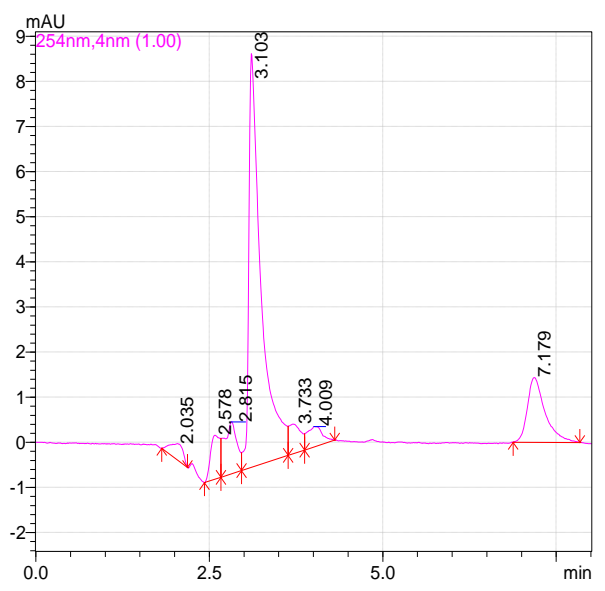

a

Figure 2. HPLC chromatograms a) MDA (retention time 7.179) b) vitamin C (retention time 4.099).
2.5. HPLC quantification of vitamin $E$ and vitamin A

Lipid soluble vitamins in cells were assayed according to the method of Catignani [29]. After incubation with the compounds in $100 \mu \mathrm{l}$ of the cells suspensions, 200 $\mu \mathrm{L}$ of Ethanol: Sulfuric acid (99: 1) and $100 \mu \mathrm{L}$ of water was added for precipitation of proteins. After thorough mixing with vortex, it was centrifuged at $4500 \mathrm{rpm}$ for 5 minutes. Then $100 \mu \mathrm{L}$ of $\mathrm{n}$-hexane ( $0.05 \%$ butylated hydroxytoluene) was added on the centrifuged samples. With the addition of hexane, the
$\mathrm{NaH}_{2} \mathrm{PO}_{4}$ and $5.2 \mathrm{mM} \mathrm{KCl}, \mathrm{pH}$ 7.4). Then the mediums were treated with $0.1 \mathrm{ml}$ of $0,5 \mathrm{M}$ perchloric acid and $0,1 \mathrm{ml}$ water. The cells were scraped from the tubes and the lysates were santrifuged $5 \mathrm{~min}$ at ambient temperature. The supernatant were taken and separated $17,5 \%$ methanol (v/v) in $30 \mathrm{M}$ monobmasic potassium phosphate buffer $(\mathrm{pH} 3,6)$ mobile phase.

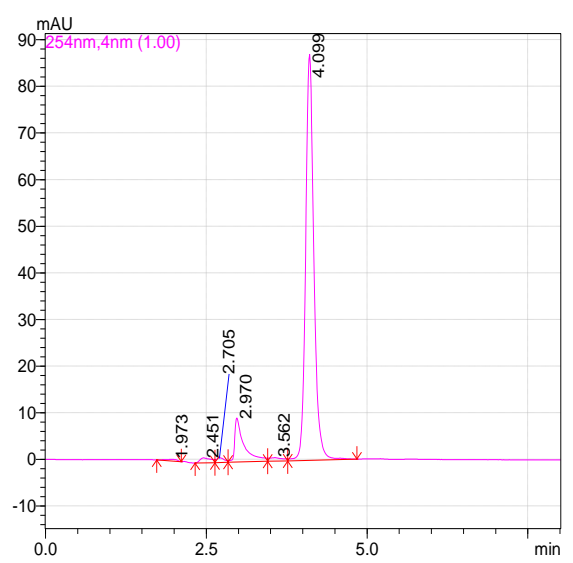

b lipid-soluble vitamins in the medium were extracted into the hexane phase. The tubes were mixed on vortex and centrifuged again. At the end of the centrifuge, the hexane phase was carefully separated and taken into the glass tube. $100 \mu \mathrm{L}$ of $\mathrm{n}$-hexane was added onto the sample again, mixed and centrifuged, and the $n$-hexane phase was combined with the hexane phase in the glass tube. The extracted hexane phase was carefully evoperated by using nitrogen gas. The residue from the hexane was dissolved in $100 \mu \mathrm{l}$ of mobile phase (methanol/acetonitrile/chloroform, 47:42:11, v/v). 20 $\mu \mathrm{L}$ of this solution was taken and injected into HPLC.
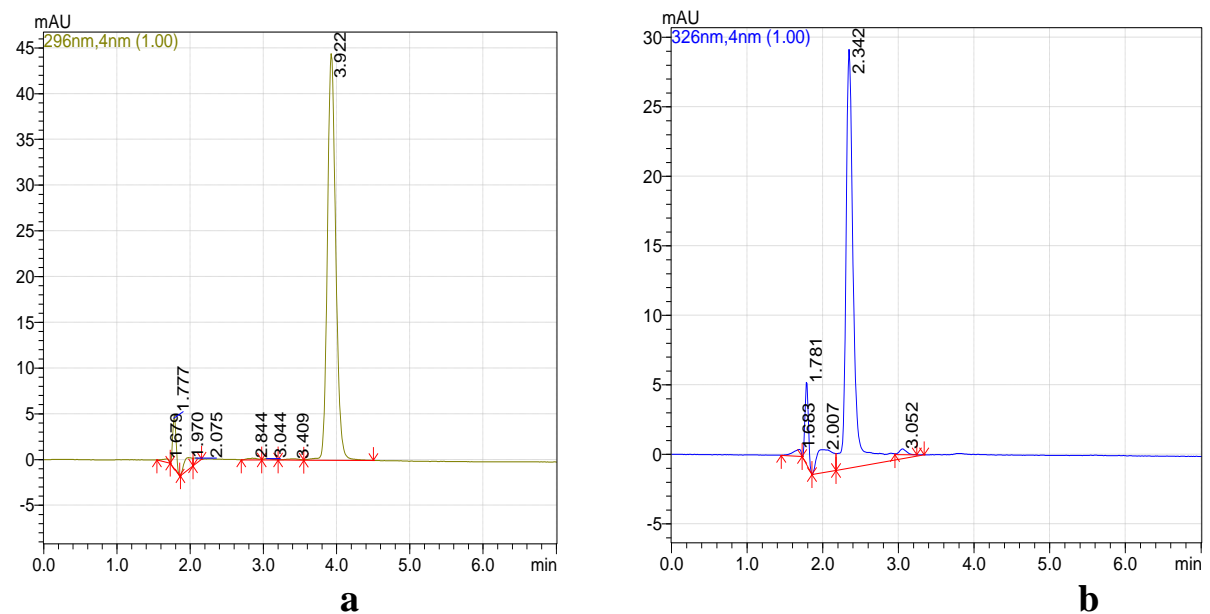

Figure 3. HPLC chromatograms a) vitamin E (retention time 3.922) b) vitamin A (retention time 2.342). 


\subsection{Apparatus}

Liquid chromatographic system consisted of LC-20AD pumps, DGU-20A5 degasser, SIL 20A autosampler, CTO-10AS VP column oven, SPD-M20A DAD system. These apparatus were connected via a communication module (Model CBM-20A), and controlled by a Shimadzu LC Solution Workstation (Shimadzu, Kyoto, Japan). A Shimadzu Shim-pack vpODS column $(150 \mathrm{~L} \times 4.6)$ was used.

\subsection{Statistical analysis}

SPSS 22.0 for Windows software (SPSS Inc., New York, IBM, USA) were used for statistical analyses. The statistical significance was set at $\mathrm{P}<0.05$ and results are expressed as mean \pm S.D. Comparison between mean values for antioxidant parameters were made by independent sample t-test.

\section{Results and Discussion}

\subsection{Selection of working type}

Only in vitro method cannot be sufficient to determine antioxidant activity, and therefore in vivo methods are required to assess antioxidant activity. Due to this fact, antioxidant activities of the new compounds have been tested by using Saccharomyces cerevisiae yeast cells as in vivo model [30].

\subsection{Induction of lipid peroxidation by test compounds}

The mean MDA levels of the groups are given in Table 1 and Figure 2a. Significant differences were found in MDA levels. After $24 \mathrm{~h}$ incubation of the Saccharomyces cerevisiae yeast cells with test compounds, the levels of MDA in L2, L3 and L8 groups statistically decreased and compound L9 induced lipid peroxidation from compared to the controls.

\subsection{Effects of the compounds on the vitamins $A$ and $\mathrm{E}$ levels of cells}

Vitamins E levels are increased by the L2 and L8 and decreased by the L9 compounds treatment (Table 1 and Figure 2b). Vitamin A and C levels were not changed all compounds statistically. The stopping chain reactions, metal ion binding activity, radical capture activity and reducing force factors explain of a possible antioxidant substance which it posses antioxidant activity [31].

This work was carried out in order to verify and to clarify the contribution of the biological side effects and relation of new compounds with reactive oxygen species (ROS). Reactive oxygen species (ROS) are formed and degraded by all aerobic organisms, leading to either physiological concentrations required for normal cell function or excessive quantities, the state called oxidative stress [32]. The malondialdehyde (MDA) [33] and isoprostanes [34], well known lipid peroxidation products, are produced ROS-mediated oxidation of membrane lipids.

Table 1. The mean values of the vitamin A, E, C and MDA of Saccharomyces cerevisiae yeast cells treated with the compounds

\begin{tabular}{lllll}
\hline Groups & \multicolumn{1}{c}{ C vitamin, ppm } & MDA, ppm & Vitamin A, ppm & Vitamin E, ppm \\
\hline & & & & \\
Control & $0.79 \pm 0.04$ & $22.24 \pm 0.57$ & $0.07 \pm 0.009$ & $0.87 \pm 0.08$ \\
L1 & $0.72 \pm 0.05$ & $21.47 \pm 1.15$ & $0.07 \pm 0.011$ & $0.74 \pm 0.05$ \\
L2 & $0.84 \pm 0.04$ & $17.34 \pm 0.88^{* *}$ & $0.08 \pm 0.003$ & $0.94 \pm 0.05^{*}$ \\
L3 & $0.69 \pm 0.06$ & $19.33 \pm 1.26^{*}$ & $0.08 \pm 0.007$ & $0.74 \pm 0.04$ \\
L4 & $0.74 \pm 0.07$ & $21.26 \pm 2.15$ & $0.07 \pm 0.005$ & $0.86 \pm 0.04$ \\
L5 & $0.74 \pm 0.06$ & $20.84 \pm 1.32$ & $0.07 \pm 0.004$ & $0.74 \pm 0.04$ \\
L6 & $0.75 \pm 0.08$ & $20.76 \pm 1.23$ & $0.07 \pm 0.006$ & $0.75 \pm 0.03$ \\
L7 & $0.75 \pm 0.07$ & $21.59 \pm 1.15$ & $0.07 \pm 0.007$ & $0.76 \pm 0.03$ \\
L8 & $0.85 \pm 0.07$ & $17.66 \pm 0.81^{* *}$ & $0.08 \pm 0.004$ & $0.96 \pm 0.05^{*}$ \\
L9 & $0.64 \pm 1.00$ & $26.40 \pm 0.94^{*}$ & $0.07 \pm 0.004$ & $0.72 \pm 0.03^{*}$ \\
L10 & $0.74 \pm 0.08$ & $21.84 \pm 0.53$ & $0.06 \pm 0.008$ & $0.79 \pm 0.04$ \\
\hline
\end{tabular}

$* \mathrm{p}<0.05 ; \quad * * \mathrm{p}<0.01 ;$ 

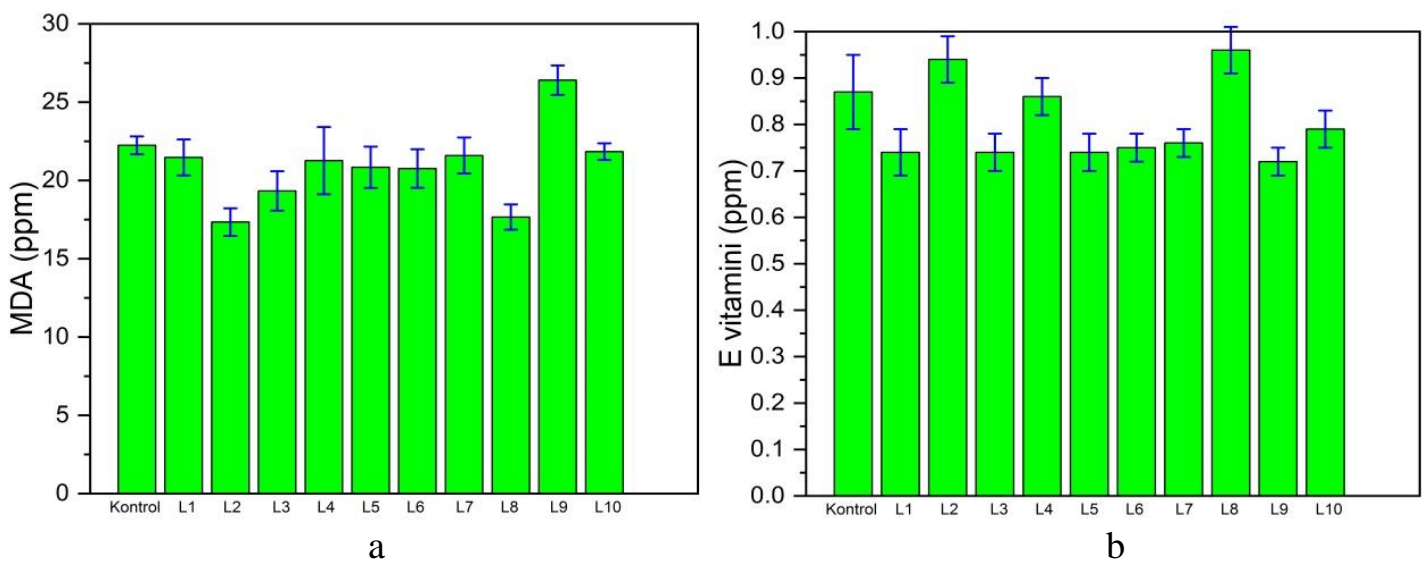

Figure 4. Comparison of the mean concentration of the a) MDA and b) vitamin $\mathrm{E}$ compound treated cells, (*p $\leq 0.05$, $* * \mathrm{p} \leq 0.01)$.

ROS are continuously produced in actively metabolizing cells. However, Saccharomyces cerevisiae, like all organisms, contains effective antioxidant defense mechanisms, which detoxify ROS as they are generated and maintain the intracellular redox environment in a reduced state. An oxidative stress is said to occur when ROS overwhelm these defenses, resulting in genetic degeneration and physiological dysfunction, leading eventually to cell death.

The cellular antioxidant systems have two major groups, enzymatic and nonenzymatic [32]. The vitamin $\mathrm{C}$, vitamin $\mathrm{E}$ and carotenoids are non enzymatic low molecular weight antioxidant compounds and they are consumed by the metabolism and may fall below normal ranges. For the evaluation of oxidative stress in biological systems, the analyses of levels of antioxidants and MDA are very important. Saccharomyces cerevisiae, as a typical eukaryotic model microorganism has some advantages in comparison to humans and animals, such as its simple structure that known genetic background and easy manipulation. Moreover, Saccharomyces cerevisiae genome possesses a strikingly high-level of functional conservation within the human genome and other higher eukaryotes. In consideration of these advantages, Saccharomyces cerevisiae has become a prominent model for evaluating some cell damages to quickly provide functional clues [30].

In order to eliminate the harmful effects of oxidative stress, many researches are carried out to illuminate the oxidation antioxidation mechanisms and produce solutions. These mechanisms are very important in various living systems, especially human beings. Yeast cells and mammalian cells largely are similar. For this reason, aging, apoptosis and various diseases caused by oxidative stress are important in lighting studies.
Cytotoxicity of xenobiotics could be explained by the impairment of either cellular regulation system, intracellular synthesis of macromolecules or cellular transduction signalling. For the determination of antioxidant and prooxidant activity of test compounds, we choose and consider MDA and antioxidant vitamins [35]. The cell death occurs as a result of membranes that change structure and lose function with lipid peroxidation and increased production of MDA [36]. MDA can be measured by many methods and it is one of the most important indicators of lipid peroxidation [37, 38]. The results show that in the Saccharomyces cerevisiae yeast cells, L9 compound induced oxidative damage by generating lipid peroxidation which it increased MDA formation and decreased antioxidant vitamin E levels. The presence of high MDA levels in sample points to the induction of peroxidation following $\mathrm{L} 9$ administration.

As seen from the table and figures, L1, L3, L4, L5, L6, L7 and L10 did not affect vitamins A, E, C and MDA levels but L2 and L8 caused significant increase vitamin $\mathrm{E}$ and the decrease MDA concentrations statistically which has not been previously described. This indicates that the L2 and L8 supplements were able to spare the vitamin E concentrations with decreasing the lipid peroxidation in the cells.

The Vitamin E antioxidant capacity is very large and high because its antioxidant duty performs by using all mechanisms such as radical destruction, breaking the chain, suppression, repairing broken structures [31, 32].

The more literatures showed that the thiadiazole nuclei have anticonvulsant, anti-leishmanial, antimicrobial, antidepressant, antioxidant, radio protective, anticancer and anti-inflammatory activities.

Kus et al [39] synthesized some novel 5-[(2(substituted phenyl)-1H-benzimidazole-1-yl)methyl]methyl-1,3,4- thiadiazole-2-amines and tested for 
antioxidant properties by using various in vitro systems. They have stated that some compounds prevented lipid peroxidation slightly at $10^{-3} \mathrm{M}$ concentration.

The 6-[3-(4-fluorophenyl)-1H-pyrazol-4-yl]-3[(2naphthyloxy)methyl] [1,2,4] triazolo [3,4-b]-[1,3,4] thiadiazole (FPNT) and 6-[3-(4chlororophenyl)-1Hpyrazol-4-yl]-3-[(phenyloxy)methyl]-[1,2,4] triazolo $[3,4 \mathrm{~b}][1,3,4]$ thiadiazole (CPPT) in vitro antioxidant activity had investigated by DPPH and ABTS radical scavenging methods as well as by lipid peroxide assay [40]. The results of these assays proved FPNT to be an excellent antioxidant.

The antioxidant activity studies of 1,3,4-thiadiazoles demonstrated that the thiol, thiosulfonic acid and phosphorothioate derivatives of thiadiazoles exhibit evident antioxidant activity. This activity can explain direct link between biological function and thiol containing aromatic ring [41].

The thiol and aminothiol derivative thiadiazoles were synthesized by the Prouillac et al [42] and examined them for their antioxidant ability. The new compounds demonstrated the most scavenging activity for $\mathrm{DPPH} \bullet$ and $\mathrm{ABTS} \bullet+$ free radicals.

\section{Conclusion}

The increase in free radical formation causes an increase in MDA level and GSH-Px enzyme activity and a decrease in vitamin $E$ level.

As a result of our experimental studies, the groups that the chemical substances used have affected their activities significantly.

In conclusion, we investigated the antioxidant and prooxidant effects of test compounds on Saccharomyces cerevisiae yeast cells and the results of this work demonstrate that L9 compound caused oxidative stress and L2 and L8 have antioxidant activity.

The antioxidant activity evaluation results have demonstrated that only aromatic groups exhibit evident antioxidant activity. It seems that our findings are compatible with the literature.

\section{Acknowledgment}

We thanks to Dr. Sevda KIRBAĞ for giving and helping for cell studies.

\section{References}

[1] Yang D., An B., Wei W., Tian L., Huang B., Wang H. Coppercatalyzed domino synthesis of nitrogen heterocycle-fused benzoimidazole and 1,2,4benzothiadiazine 1,1-dioxide derivatives, A C S Comb Sci., 17 (2015) 113-119.

[2] Iizawa, Y., et al. Therapeutic effect of cefozopran
(SCE-2787), a new parenteral cephalosporin, against experimental infections in mice, Antimicrobial Agents and Chemotherapy 37 (1993) 100-105.

[3] Oruc, E.E., et al. 1,3,4-thiadiazole derivatives. Synthesis, structure elucidation, and structureantituberculosis activity relation- ship investigation, Journal of Medicinal Chemistry. 47 (2004) 6760-6767.

[4] Foroumadi, A., et al. Antituberculosis agents VIII. Synthesis and in vitro antimycobacterial activity of alkyl alpha-[5-(5-nitro-2-thienyl)-1,3,4thiadiazole-2-ylthio]acetates, Farmaco, 58 (2003) 1073-1076.

[5] Kamal M.D., et al. Synthesis, anticonvulsant, and anti-inflammatory evaluation of some new benzotriazole and benzofuran-based heterocycles, Bioorganic and Medicinal Chemistry, 14 (2006) 3672-3680.

[6] Mullick P., et al. Thiadiazole derivatives as potential anticonvulsant agents, Bulletin of the Korean Chemical Society, 32 (2011) 1011-1016.

[7] Clerici, F., et al. Synthesis of 2-amino-5-sulfanyl1,3,4-thiadiazole derivatives and evaluation of their antidepressant and anxi-olytic activity, Journal of Medicinal Chemistry 44 (2001) 931-936.

[8] Hasui,T., et al. Identification of benzoxazin-3-one derivatives as novel, potent, and selective nonsteroidal mineralocorticoid receptor antagonists, Journal of Medicinal Chemistry, 54 (2011) 8616-8631.

[9] Zheng, K. B., et al. Synthesis and antitumor activity of N1-acetylamino-(5-alkyl/aryl-1,3,4-thiadiazole2-yl)-5-fluorouracil derivatives, Chinese Chemical Letters, 19 (2008) 1281-1284.

[10] Chen, C.J., et al. Synthesis and antifungal activities of 5-(3,4,5-trimethoxyphenyl)-2sulfonyl-1,3,4thiadiazole and 5-(3,4,5- trimethoxyphenyl)-2sulfonyl-1,3,4-oxadiazole derivatives, Bioorganic\& Medicinal Chemistry, 15 (2007) 3981-3989.

[11] Suzuki, F., Kawakami, I., Yamamoto, S., Kosai, Y. Japan Kokai, 7776432 1977; Chem. Abstr., 88 (1978) 100351

[12] Abdel-Ramhan A. E., Mahmoud A. M., El-Sherief H.A., Gahatta A.G., Chem. Abstr., 98 (1983) $72012 b$.

[13] Foerster H., Mues V., Baasner B., Hagemann H., Eue I., Schmidt R. EuropeanPatent, 60426 1981; Chem. Abstr., (1983) 72107m.

[14] Tiwari N., Chaturvedi B., Nizamuddin A. ynthesis and fungicidal activities of some 2-aryloxymethyl1,3,4-thiadiazolo[2,3,-b]-quinazolin-4-one and 2aryloxymethyl-5-substituted-1,3,4-

thiadiazolo[3,2,-a]-1,3,5-triazine-7-thiones, Indian 
J. Chem., 28 (1989) 200-202.

[15] Singh H., Yadav L.D.S., Agri. Biol. Chem., 40 (1976) 759-764; (b) Chaaban I., Oji O.O.J. Indian Chem Soc., 61 (1984) 523-525; (c) Mohsen A., Omer M.E., Aboulwafa O.M.J. Heterocycl. Chem., 21 (1984) 1415-1418; (d) Hiremath S.P., Birador J.S., Kudari S.M.J., Indian Chem. Soc., 61 (1984) 74-76.

[16] Rao A. V. R., Bose D.S., Gurjar M.K., Ravindranatran T. Tetrahedron, 45 (1989) 70317040; (b) Seydenpenn J. Chiral Auxiliaries and Ligands in Asymmetric Synthesis, John Wiley: New York, (1995); (c) Wright A.E., Schafer M., Midland S., Munnecke D.E., Sims J.J., Tetrahedron Lett., 30 (1989) 5699-5702.

[17] Kolb H.C., Sharpless K.B., A simplified procedure for the stereospecific transformation of 1,2-diols into epoxides, Tetrahedron, 48 (1992) 1051510530.

[18] Lohray B.B., Ahuja J.R., J. Chem. Soc. (1991) 9597; (b) Nicolaou K.C., Huang X., Snyder S.A., Rao P.B., Bela M., Reddy M.V., Angew., Chem. Int., 41 (2002) 834.

[19] Parida S., Dordick J.S.J. Am. Chem. Soc., 113 (1991) 2253-2259; (b) Nelson W.L., Wennerstrom J.E., Sankar S.R.J. Org. Chem., 42 (1977) 10061012; (c) Bian chi D., Bosetti A., Cesti P., Golini,P.Tetrahedron Lett., 33 (1992) 3231-3234.

[20] Kurina-Sanz M., Bisogno F.R., Lavandera I., Orden A.A., Gotor V., Promiscuous substrate binding explains the enzymatic stereo- and regiocontrolled synthesis of enantiopure hydroxy ketones and diols, Adv. Synth. Catal., 351 (2009) 1842- 1848.

[21] Supuran C.T., Scozzafava A., Carbonic anhydrase inhibitors, Curr Med Chem Immunol Endocrinol Metab Agents, 1 (2001) 61-97.

[22] Singh, N., Rajini, P. S., Free radical scavenging activity of an aqueous extract of potato peel, Food Chem, 85 (2004) 611.

[23] Prior, R. L., Wu, X., Schaich, K., Standardized Methods for the Determination of Antioxidant Capacity and Phenolics in Foods and Dietary Supplements, J. Agric. Food Chem., 53 (2005) 4290.

[24] Uchida, K., Role of Reactive Aldehyde in Cardiovascular Diseases, Free Radical Biol. Med., 28 (2000) 1685.

[25] Cadenas, E., Davies, K. J. A., Mitochondrial Free Radical Generation, Oxidative Stress, and Aging, Free Radical Biol. Med., 29 (2000) 222.

[26] Offeing, B.M., Martelli, S.,. Steochemistry and Antitumour Activity of Platinium Metal Complexes of 2- Acetypyridine Thiosemicarbazones, Transition Metal Chemistry., 22 (1997) 263-269.

[27] Karatepe A., (1r, 2r)-etan-1,2 diol tiyadiyazol türevlerinin sentezi, karakterizasyonu ve biyolojik özellikleri. Doktora tezi, Bingöl, 2020.

[28] Karatepe, M., Simultaneous Determination of Ascorbic Acid and Free Malondialdehyde in Human Serum by HPLC/UV. LC-GC, North America,. 22 (2004) 362-5.

[29] Catignani, G.L., Simultancous Determination of Retinol and $\alpha$-Tocopherol in Serum of Plazma by Liquid Chromatography, Clin. Chem, 2914 (1983) 708-712.

[30] Almeida, G.M., Thomazellab, D.P.T., Pereira, G.A.G., Monteiro G., Heterologous expression of an alternative oxidase from Moniliophthora perniciosa in Saccharomyces cerevisiae: Antioxidant function and in vivo platform for the study of new drugs against witches' broom disease, Fungal Genetics and Biology, 126 (2019) 50-55.

[31] Yildirim A., Mavi A., Kara A.A., Determination of antioxidant and antimicrobial activities of Rumexcrispus L. extracts, J. Agri. Food. Chem., 49 (2001) 4083 .

[32] Nordberg, J., Arner, E.S.J., , Reactive Oxygen Species, Antioxidants and The Mammalian Thioredoxin System, Free Rad. Biol. and Med., 31(11) (2001) 1287-1317.

[33] Maccarrone, M (Maccarrone, M); Catani, MV (Catani, MV); Iraci, S (Iraci, S); Melino, G (Melino, G); Agro, AF (Agro, AF) A survey of reactive oxygen species and their role in dermatology, J. of the Eu. Academy of Der. and Ven., 8 (1997) 185202.

[34] Morrow J.D., The isoprostanes: their quantification as an index of oxidant stress status in vivo, Drug Metab Rev., 32 (2000) 377-85.

[35] Vaca, C.E., Wilhelm J., Ringdahl M. H., Interaction of lipid peroxidation products with DNA. A review, Mutation Res., 195 (1988) 137-149.

[36] EnnamanyaJ.P., LavergnebJ.P., Reboudb G., Mode of action of bolesatine, a cytotoxic glycoprotein from Boletus satanas Lenz., Mechanistic approaches Toxicolog, 100 (1995) 51-55.

[37] Bird, R.P., Draper, H.H., Comperative Studieson Differant Methods of Malondialdehyde Determination, Methods in Enzymology., 105 (1984) 299-305.

[38] Tomita M., Okuyama T., Kawai S.J., Determination of malonaldehyde in oxidized biological materials by high-performance liquid chromatography, Chromatogr, 31 (1990) 391-7.

[39] Kus C., Kilcigil G.A., Ozbey S., Kaynak F.B., Kaya M., Coban T., Eke B.,C., Synthesis and Antioxidant Properties of Novel N-Methyl-1,3,4-Thiadiazol-2Amine and 4-Methyl-2H-1,2,4-Triazole-3(4H)Thione Derivatives of Benzimidazole Class Bioorg, Med. Chem., 16 (2008) 4294-4303. 
[40] Dhanya S., Arun M.I., Prakash S., et al. 6-[3-(4Fluorophenyl)-1H-pyrazol-4-yl]-3-[(2naphthyloxy)methyl] $[1,2,4]$ triazolo[3,4b] $[1,3,4]$ thiadiazole as a potent antioxidant and an anticancer agent induces growth inhibition followed by apoptosis in HepG2 cells, Arabian Journal of Chemistry, 3 (2010) 211-217.

[41] Cressier D., Prouillac C., Hernandez P., et al., Synthesis, Antioxidant Properties and
Radioprotective Effects of New Benzothiazoles and Thiadiazoles, Bioorg. Med. Chem., 17 (2009) 52755284.

[42] Prouillac C., Vicendo P., Garrigues J.C., Poteau R., Rima G., Evaluation of new thiadiazoles and benzothiazoles as potential radioprotectors: free radical scavenging activity in vitro and theoretical studies (QSAR, DFT), Free Rad Biol Med., 46 (2009) 1139-1148 